\title{
DETERMINAÇÃO DA CONDUTIVIDADE HIDRÁULICA E DA SORVIDADE DE UM SOLO NÃO-SATURADO UTILIZANDO-SE PERMEÂMETRO A DISCO1
}

\author{
EDVANE BORGES ${ }^{2}$, ANTONIO CELSO DANTAS ANTONINO ${ }^{3}$, ATTILIO DALL'OLIO $^{4}$, \\ PIERRE AUDRY5 ${ }^{5}$ CLEMENTE JOSÉ GUSMÃO CARNEIRO ${ }^{6}$
}

\begin{abstract}
RESUMO - Dois métodos, utilizando permeâmetros a disco, foram usados para medir a sorvidade e a condutividade hidráulica em três horizontes de um solo de Santa Maria da Boa Vista, Pernambuco, utilizando-se potenciais de fornecimento de água de $0,-2,5,-5$ e $-10 \mathrm{~cm}$ de água. No primeiro método, a condutividade hidráulica foi obtida através de estimativas da sorvidade e do fluxo estacionário, utilizandose apenas um permeâmetro. No segundo, as medidas da sorvidade e da condutividade hidráulica foram feitas utilizando-se valores dos fluxos estacionários, obtidos a partir de dois permeâmetros de diferentes raios. Esse segundo método apresentou resultados mais consistentes dos tempos - gravitacional e geométrico -, e do raio característico de poros que o primeiro método. A sorvidade foi tão importante quanto a condutividade hidráulica para caracterizar o processo de infiltração. A redução brusca dos valores dos raios característicos de poros do horizonte $\mathrm{A} 2 / \mathrm{B}$, com mudanças no potencial de fornecimento de água, revelou a heterogeneidade da estrutura deste horizonte, permitindo identificar a influência dos volumes argilosos compactados sobre o processo de infiltração.
\end{abstract}

Termos para indexação: permeâmetro, infiltração.

\section{DETERMINATION OF UNSATURATED HYDRAULIC CONDUCTIVITY AND SORPTIVITY OF A SOIL USING A DISK PERMEAMETER}

\begin{abstract}
Sorptivity and hydraulic conductivity were determined by two methods using disc permeameters. Infiltration experiments were carried out on three horizons of a representative soil of the semi-arid region, located in Santa Maria da Boa Vista, State of Pernambuco, Brazil. Water was supplied to this soil by disc permeameters at potentials of $0,-2.5,-5$ and $-10 \mathrm{~cm}$ of water. In the first method, hydraulic conductivity was measured using estimates of sorptivity and steady state fluxes. In the second, sorptivity and hydraulic conductivity were determined using measurements of steady state fluxes with two disc permeameters of different radius. Results of the second method, regarding characteristic times and characteristic pore radius, were more consistent than those of the first one. Sorptivity was as important as hydraulic conductivity to characterize the infiltration process. The abrupt reduction of the characteristic pore radius in the horizon $\mathrm{A} 2 / \mathrm{B}$ with changes in the water supply potential indicates the structure heterogeneity of this horizon and allows the identification of the role of the clay compact volumes on the infiltration process.
\end{abstract}

Index terms: permeameter, infiltration.

\footnotetext{
${ }^{1}$ Aceito para publicação em 4 de maio de 1999.

${ }^{2}$ Físico, M.Sc., Dep. de Energia Nuclear (DEN), Universidade Federal de Pernambuco (UFPE), Av. Prof. Luiz Freire, 1000, CEP 50540-740 Recife, PE.

${ }^{3}$ Eng. Civil, Ph.D., Prof. Adjunto, UFPE. E-mail 02acda@npd.ufpe.br

${ }^{4}$ Físico, M.Sc., Prof. Adjunto, UFPE. E-mail: ado@elogica.com.br

${ }^{5}$ Pedologista, Convênio ORSTOM/CNPq, UFPE. E-mail pierreaudr@elogica.com.br
}

\section{INTRODUÇÃO}

O processo de infiltração é caracterizado por dois parâmetros: a sorvidade $\left(\mathrm{S}_{0}\right)$ e a condutividade hidráulica $\left(\mathrm{K}_{0}\right)$. $\mathrm{O}$ valor de $\mathrm{S}_{0}$ é associado à fase inicial da infiltração. Nessa fase, a infiltração é praticamente independente dos efeitos gravitacionais

\footnotetext{
${ }^{6}$ Eng. Elétr., Ph.D., Prof. Titular, UFPE. E-mail cjsilva@elogica.com.br
} 
e geométricos. Os valores $\mathrm{S}_{0}$ e $\mathrm{K}_{0}$ na camada superficial do solo definem a partição dos aportes externos de água (precipitação, irrigação), afetando assim tanto o armazenamento de água no solo, como o escoamento superficial. É importante considerar que os macroporos são funcionais quando o solo está muito próximo à saturação, sendo capazes de transportes preferenciais de água e substâncias químicas de forma rápida durante o processo de infiltração. Por causa da natureza frágil dos macroporos é necessária a realização de medidas de infiltração in situ usando técnicas que minimizem as perturbações do solo. Do ponto de vista mais prático, pode-se avaliar a importância dos macroporos sobre a infiltração em condições de inundação (Watson \& Luxmoore, 1986) através da diferença entre $S_{0}$ e $K_{0}$ a um potencial matricial $\psi_{0}$, próximo à saturação, e $\mathrm{Se} \mathrm{K}$ medidos à saturação.

O processo de infiltração pode ser estudado utilizando-se dispositivos chamados permeâmetros a disco. Técnicas baseadas nesses permeâmetros são de grande interesse, pois permitem determinar simultaneamente, $\mathrm{S}_{0}$ e $\mathrm{K}_{0}$ in situ, de forma simples e rápida, minimizando as perturbações no solo. A análise das relações entre $\mathrm{S}_{0}$ e $\mathrm{K}_{0}$ pode ser usada para inferir a importância relativa das forças capilares, da gravidade e da geometria da fonte de água em controlar a densidade de fluxo de água que entra no solo, permitindo caracterizar o seu comportamento hidráulico. A baixa penetração da frente de umedecimento permite caracterizar o solo em pequenas espessuras (camadas de superfície, interfaces entre dois horizontes).

Inicialmente, os permeâmetros a disco foram usados para medir as taxas de infiltração mantendo-se um potencial positivo e constante na superfície do solo (Talsma, 1969). Posteriormente, foram desenvolvidos dispositivos que permitem manter esse potencial constante e negativo (Dirksen, 1975; Clothier \& White, 1981), possibilitando a determinação das propriedades físico-hídricas de solos fissurados. Perroux \& White (1988) aperfeiçoaram esse dispositivo, a fim de se obter um maior controle do potencial negativo mantido na base do disco.

Dois métodos são usados para determinar $\mathrm{S}_{0} \mathrm{e} \mathrm{K}_{0}$ a partir de medidas das taxas de infiltração, obtidas com permeâmetros a disco, tanto nos instantes inici- ais desse processo, onde se aplica a teoria de Philip (1969), como no regime estacionário, empregando a equação proposta por Wooding (1968). O primeiro método de Perroux \& White (1988) é baseado no uso de um único permeâmetro, e requer estimativas da sorvidade e do fluxo estacionário. $\mathrm{O}$ fato de esse método estimar a sorvidade, a partir do comportamento inicial da infiltração acumulada na razão da raiz quadrada do tempo, representa uma desvantagem na obtenção deste parâmetro, sobretudo em solos úmidos e altamente permeáveis. O segundo, de Smettem \& Clothier (1989), necessita dos valores dos fluxos estacionários, medidos a partir de dois permeâmetros de raios diferentes situados em locais tão próximos quanto possível, visando diminuir o efeito da variabilidade espacial do solo.

O objetivo deste trabalho foi comparar dois métodos de determinação dos valores de sorvidade e de condutividade hidráulica, com o uso de permeâmetros a disco, em três horizontes de um solo do Sertão do Estado de Pernambuco.

\section{MATERIAL E MÉTODOS}

\section{Teoria}

No início da infiltração, quando a capilaridade é dominante, tem-se que a infiltração acumulada em função do tempo (I(t)) é determinada pela equação (Philip, 1957):

$\mathrm{I}=\mathrm{S}_{0} \mathrm{t}^{1 / 2}$

onde $\mathrm{S}_{0}$ representa a sorvidade $\left(\mathrm{cm} / \mathrm{s}^{1 / 2}\right)$.

De acordo com Wooding (1968), o fluxo de água que passa através do permeâmetro a disco de raio $r$, tende para o valor estacionário:

$$
\begin{aligned}
& \mathrm{q}_{\infty}=\mathrm{K}_{0}+\frac{4 \phi_{0}}{\pi} \frac{1}{\mathrm{r}} \\
& \text { onde } \phi_{0}=\int_{\psi_{\mathrm{n}}}^{\psi_{0}} \mathrm{~K}(\psi) \mathrm{d} \psi=\int_{\theta_{\mathrm{n}}}^{\theta_{0}} \mathrm{D}(\theta) \mathrm{d} \theta
\end{aligned}
$$

é a equação transformada de Kirchoff, ou o potencial de fluxo matricial (Gardner, 1958); $\mathrm{K}_{0}$, a condutividade hidráulica correspondente ao potencial da água $\psi_{0}, \psi_{\mathrm{n}}, \mathrm{o}$ potencial da água do solo antes de ser iniciado o experimento, e $\theta_{\mathrm{n}}$ e $\theta_{0}$ as umidades volumétricas inicial e final.

Assumindo-se a expressão exponencial para $\mathrm{D}(\theta)$, relacionada com a sorvidade $\mathrm{S}_{0}$, sugerida por Reichardt et al. (1972): 
$D(\theta)=\frac{\gamma S_{0}^{2}}{\left(\theta_{0}-\theta_{n}\right)^{2}} \exp \left[\frac{\beta\left(\theta-\theta_{n}\right)}{\left(\theta_{0}-\theta_{n}\right)}\right]$

onde $\beta$ e $\gamma$ são parâmetros que podem ser obtidos através de ajustes por mínimo quadrado, e integrando-se a Equação 3, obtém-se

$\phi_{0}=\left(\begin{array}{c}\mathrm{bS}_{0}^{2} \\ \left(\theta_{0}-\theta_{\mathrm{n}}\right.\end{array}\right)$

$\operatorname{com} b=\gamma\left(\frac{\exp (\beta)-1}{\beta}\right)$

onde b é um parâmetro de forma da difusividade $(1 / 2 \leq b \leq \pi / 4)$, que foi considerado igual a 0,55 (White \& Sully, 1987)

Durante o processo de infiltração tridimensional, os fatores que podem afetar o fluxo de entrada de água no solo são: a geometria da fonte de água, a capilaridade e a gravidade. Esses fatores são caracterizados pelas escalas de tempo - gravitacional ( $\left.\mathrm{t}_{\text {grav }}\right)$ e geométrico $\left(\mathrm{t}_{\text {geom }}\right)$ - e de comprimento - comprimento capilar $\left(\lambda_{\mathrm{c}}\right)$ e raio característico de poros $\left(\lambda_{\mathrm{m}}\right)$.

Os tempos gravitacional e geométrico são determinados respectivamente pelas equações (Thony et al., 1991):

$$
\begin{aligned}
& \mathrm{t}_{\text {grav }}=\left(\frac{\mathrm{S}_{0}}{\mathrm{~K}_{0}}\right)^{2} \\
& \mathrm{t}_{\text {geom }}=\left(\frac{\mathrm{r}\left(\theta_{0}-\theta_{\mathrm{n}}\right)}{\mathrm{S}_{0}}\right)^{2}
\end{aligned}
$$

O tempo gravitacional pode ser interpretado como o intervalo durante o qual a dinâmica da infiltração é controlada mais pelas forças capilares do que pela ação da gravidade; enquanto o tempo geométrico representa o intervalo durante o qual o caráter unidimensional do processo de infiltração não é alterado. Talsma (1969) observou que a influência da gravidade pode ser considerada desprezível para $t \leq 0,02 t_{\text {grav }}$. Em estudos realizados no campo, foram obtidos valores de $t_{\text {grav }}$ variando entre 0,08 a 34 horas (White \& Sully, 1987). Segundo Philip (1969), o intervalo de tempo no qual a Equação 1 pode ser aplicada deve ser menor que $t_{\text {geom. }}$.

A escala de comprimento capilar é dada por White \& Sully(1987):

$$
\lambda_{\mathrm{c}}=\frac{\mathrm{bS} S_{0}^{2}}{\left(\theta_{0}-\theta_{\mathrm{n}}\right) \mathrm{K}_{0}}
$$

Essa escala representa a importância relativa das forças capilares em relação à gravidade, quando a água é transmitida de uma fonte mantida a $\theta_{0}$ através do solo, com umidade inicial $\theta_{\mathrm{n}}$.

A partir da teoria da capilaridade, Philip (1987) definiu um raio característico de poros:

$\lambda_{\mathrm{m}}=\frac{\sigma}{\rho g \lambda_{\mathrm{c}}}=\frac{13,5\left(\theta_{0}-\theta_{\mathrm{n}}\right) \mathrm{K}_{0}}{\mathrm{~S}_{0}^{2}}(\mathrm{~mm})$

onde $\sigma$ é a tensão superficial, $\rho$ é a massa específica da água e $g$ a aceleração da gravidade. O raio característico de poros define a dimensão média dos poros que participam do processo de infiltração submetido à pressão aplicada $\psi_{0}$. Quanto maior for o raio característico, maior será o efeito da gravidade em comparação ao da capilaridade.

\section{Experimentos}

Os experimentos de infiltração foram realizados utilizando dois permeâmetros, P1 e P2, de concepção idêntica, representados esquematicamente na Fig. 1, diferindo apenas com relação às dimensões de suas bases, $25 \mathrm{~cm}$ e $8 \mathrm{~cm}$ de diâmetro, respectivamente.

O modo de operação foi o mesmo para os dois permeâmetros. O reservatório lateral foi preenchido com água, através do tubo $\mathrm{C}_{1}$, utilizando-se uma seringa hipodérmica. A pressão imposta $\psi_{0}$ é dada pela diferença entre $\mathrm{z}_{2}$ e $\mathrm{z}_{1}$ (Fig. 1), de tal forma que

$\Psi_{0}=\mathrm{z}_{2}-\mathrm{z}_{1}$

A altura $\mathrm{Z}_{2}$ é fixa, sendo de $1 \mathrm{~cm}$ para $\mathrm{P} 1 \mathrm{e} 5 \mathrm{~cm}$ para P2.

Para o preenchimento do reservatório de alimentação, a base do permeâmetro a disco foi imergida em uma bacia cheia de água e em seguida foi feita a aspiração do ar pelo tubo acoplado na rolha de borracha $\mathrm{B}_{0}$ (Fig. 1). Quando o nível da água alcançou o topo do reservatório, a pinça de Mohr (PM) foi fechada. O ar que permaneceu na base do disco foi retirado com movimentos que permitiam que as bolhas escoassem pelo reservatório de alimentação $\left(\mathrm{R}_{\mathrm{A}}\right)$. A leitura da variação do nível de água no reservatório de alimentação em determinados intervalos de tempo, permitiu a obtenção da infiltração acumulada. As observações foram interrompidas quando a variação do nível de água no reservatório, em função do tempo, tornou-se constante, ou seja, quando o regime estacionário foi alcançado.

Inicialmente, as superfícies escolhidas para a realização dos experimentos foram limpas, para retirar qualquer tipo de vegetação presente, e niveladas, eliminando assim as irregularidades mais proeminentes. Para uma adequada conexão entre a base do permeâmetro e a superfície do solo, foi necessária a colocação de um material de contato. 
Neste caso, utilizou-se uma fina camada ( $5 \mathrm{~mm}$ ) de areia, umedecida a um teor volumétrico de água de 3\% a 5\%, como sugerido por Thony et al. (1991), e distribuída uniformemente na superfície do solo. Para a determinação da umidade inicial $\left(\theta_{\mathrm{i}}\right)$ foram coletadas amostras de solo a uma distância de aproximadamente $30 \mathrm{~cm}$ da borda do permeâmetro. Para a determinação de $\theta_{0}$, amostras do solo úmido foram coletadas, imediatamente após a remoção do permeâmetro e da camada de areia, no próprio local onde estava assentado o permeâmetro.

As medidas para determinação de $\mathrm{S}_{0}$ e $\mathrm{K}_{0}$ foram efetuadas em um solo situado no município de Santa Maria da Boa Vista, Sertão de Pernambuco. O solo é classificado como Podzólico "intergrade" planossolo, e apresenta uma sucessão de três horizontes, que possuem, respectivamente, as seguintes características: A, humífero, arenoso e homogêneo; B, de tipo Podzólico, argilo-arenoso, com estrutura poliédrica fina, pouco desenvolvida, homogêneo; e

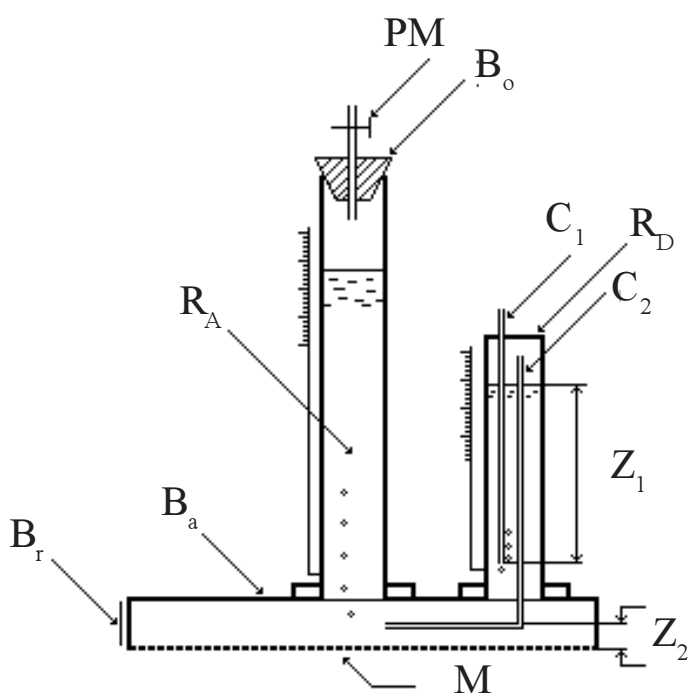

FIG. 1. Esquema do permeâmetro a disco. $B_{a}$, base de contato com o solo; $M$, membrana constituída por uma tela de tecido de náilon (abertura $20 \mu \mathrm{m}$ ) presa por um bracelete de borracha $\left(B_{r}\right) ; R_{A}$, reservatório de alimentação fechado no seu topo por uma rolha de borracha $\left(B_{0}\right)$ na qual está acoplado um pequeno tubo munido de um duto flexível e de uma pinça de Mohr (PM); $\mathbf{R}_{\mathrm{D}}$, reservatório lateral de despressurização; $C_{1}$, tubo capilar para regular o valor do potencial de fornecimento de água ao nível de membrana; $\mathrm{C}_{2}$, que realiza a comunicação entre este reservatório e a base do permeâmetro.

Pesq. agropec. bras., Brasília, v.34, n.11, p.2083-2089, nov. 1999
A2/B, profundo, também globalmente argilo-arenoso, mas de estrutura heterogênea, constituída de volumes mais argilosos, compactos e freqüentemente endurecidos (B), inseridos em uma rede arenosa, porosa, lavada e embranquecida, sem consistência (A2). Os percentuais de areia, silte e argila nesses horizontes são apresentados na Tabela 1 .

Nas profundidades de 10, 70 e $180 \mathrm{~cm}$, consideradas representativas de cada horizonte, foram realizados experimentos de infiltração com a água a potenciais que variaram de 0 até $-10 \mathrm{~cm}$. Para a determinação de $\mathrm{S}_{0}$ e de $\mathrm{K}_{0}$, em cada uma dessas profundidades, foram utilizados tanto o método de Perroux \& White (1988), como o de Smettem \& Clothier (1989). No primeiro método, o valor de $S_{0}$ foi estimado pelo ajuste, pelo método dos mínimos quadrados, da Equação 1. No segundo caso, $\mathrm{K}_{0}$ e $\mathrm{S}_{0}$ foram determinados através das Equações 12 e 13, resultantes da resolução simultânea de duas equações da forma da Equação 2, a partir das medidas dos fluxos estacionários $\mathrm{q}_{1} \mathrm{e} \mathrm{q}_{2}$ em cada permeâmetro:

$$
\begin{aligned}
\mathrm{K}_{0} & =\frac{\mathrm{q}_{1} \mathrm{r}_{1}-\mathrm{q}_{2} \mathrm{r}_{2}}{\mathrm{r}_{1}-\mathrm{r}_{2}} \\
\mathrm{~S}_{0} & =\left[\frac{\pi \mathrm{r}_{1} \mathrm{r}_{2} \Delta \theta\left(\mathrm{q}_{1}-\mathrm{q}_{2}\right)}{2\left(\mathrm{r}_{2}-\mathrm{r}_{1}\right)}\right]^{1 / 2}
\end{aligned}
$$

\section{RESULTADOS E DISCUSSÃO}

A Tabela 2 apresenta os valores de $\mathrm{S}_{0}, \mathrm{~K}_{0}, \lambda_{\mathrm{m}}$ $\mathrm{t}_{\text {grav }}$ e $\mathrm{t}_{\text {geom }}$ obtidos nas três profundidades do solo, consideradas representativas dos horizontes A, B e A2/B, pelos métodos de Perroux \& White (PW) e Smettem e Clothier (SC), nos diferentes potenciais de infiltração $\left(\psi_{0}\right)$ aplicados. Em relação ao método SC, quando os valores de $\psi_{0}$ foram mais negativos, observou-se uma diminuição da condutividade hidráulica, da sorvidade e do raio característico de poros em cada uma dessas profundidades. No método PW, observou-se um aumento dos valores dos raios característicos de poros quando os valores de $\psi_{0}$ tornaram-se mais negativos; este resultado é fisicamente incoerente pois os poros de maiores dimensões participam mais do processo de infiltração quanto menos negativo for o potencial $\psi_{0}$. Por outro lado, os resultados do método SC apresentam correta relação entre os raios 
característicos de poros e o potencial $\psi_{0}$, sugerindo a adoção preferencial deste último método. Além disso, a análise comparativa entre os valores de $\mathrm{S}_{0}$ e $\mathrm{K}_{0}$ mostra que no método SC existem relações monótonas crescentes, de $\mathrm{K}_{0}$ em relação a $\mathrm{S}_{0}$, bem definidas em todos os horizontes, enquanto no método PW tais relações são menos claras e até em sentido oposto. O horizonte $\mathrm{A} 2 / \mathrm{B}$ apresentou um comportamento diferenciado em relação aos horizontes A e B.

O método SC permitiu evidenciar, para cada horizonte, uma boa variação na condutividade hidráulica nas proximidades imediatas da saturação (Tabela 2). Por exemplo, para o horizonte A a condutividade hidráulica à saturação $\left(\psi_{0}=0\right)$ foi da ordem de nove vezes maior que a condutividade hidráulica para $\psi_{0}=-10,0 \mathrm{~cm}$
Os valores dos raios característicos de poros obtidos pelo método SC variaram de 0,31 a $0,99 \mathrm{~mm}$ nos três horizontes estudados. Esses valores estão praticamente na faixa entre $0,10 \mathrm{e}$ $0,90 \mathrm{~mm}$, que corresponde aos valores obtidos por outros autores (White \& Sully, 1987; Sauer et al., 1990; Thony at al., 1991; Cook \& Boeren, 1994).

Em virtude dos altos valores de $t_{\text {grav }}$ nas profundidades de 10 e $70 \mathrm{~cm}$, pode-se afirmar que, neste solo, a sorvidade é tão importante quanto a condutividade hidráulica para caracterizar o processo de infiltração. Considerando-se que, para um experimento de infiltração vertical, o regime estacionário quase sempre é obtido após oito a dez vezes o $t_{\text {grav }}$, seria necessário prosseguir o experimento durante várias horas e mesmo alguns dias para obter a estabilização do fluxo e, portanto, a condutividade

TABELA 1. Análise granulométrica e densidade global dos três horizontes de um solo Podzólico "intergrade" planossolo do Sertão de Pernambuco.

\begin{tabular}{|c|c|c|c|c|c|}
\hline Horizonte & $\begin{array}{l}\text { Prof. } \\
(\mathrm{cm})\end{array}$ & $\begin{array}{c}\text { Dens. global } \\
\left(\mathrm{g} / \mathrm{cm}^{3}\right)\end{array}$ & Areia & $\begin{array}{l}\text { Silte } \\
-(\%)-\end{array}$ & Argila \\
\hline A & $0-10$ & 1,61 & 91,7 & 1,6 & 6,7 \\
\hline B & $10-70$ & 1,60 & 75,4 & 1,5 & 23,1 \\
\hline $\mathrm{A} 2 / \mathrm{B}$ & $70-180$ & 1,71 & 72,5 & 3,3 & 24,2 \\
\hline
\end{tabular}

TABELA 2. Valores da variação de umidade $(\Delta \theta)$, sorvidade $\left(\mathbf{S}_{\mathbf{0}}\right)$, condutividade hidráulica do solo não-saturado $\left(K_{0}\right)$, raio característico de poros $\left(\lambda_{m}\right)$, tempos gravitacional $\left(t_{\text {grav }}\right)$ e geométrico $\left(t_{\text {geom }}\right)$, pelos métodos de Perroux \& White (PW) e Smettem \& Clothier (SC), sob alguns potenciais de fornecimento de água $\left(\psi_{0}\right)$.

\begin{tabular}{|c|c|c|c|c|c|c|c|c|c|c|c|c|}
\hline \multirow[t]{2}{*}{$\begin{array}{l}\text { Hori- } \\
\text { zonte }\end{array}$} & \multirow{2}{*}{\multicolumn{2}{|c|}{$\begin{array}{cc}\psi_{0} & \Delta \theta \\
(\mathrm{cm}) & \left(\mathrm{cm}^{3} / \mathrm{cm}^{3}\right)\end{array}$}} & \multicolumn{2}{|c|}{$\begin{array}{c}\mathrm{S}_{0} \\
\left(10^{-2} \mathrm{~cm} / \mathrm{s}^{1 / 2}\right)\end{array}$} & \multicolumn{2}{|c|}{$\begin{array}{c}\mathrm{K}_{0} \\
\left(10^{-4} \mathrm{~cm} / \mathrm{s}\right)\end{array}$} & \multicolumn{2}{|c|}{$\begin{array}{c}\lambda_{\mathrm{m}} \\
(\mathrm{mm})\end{array}$} & \multicolumn{2}{|c|}{$\begin{array}{c}\operatorname{tgrav}_{\text {gran }} \\
(\mathrm{min})\end{array}$} & \multicolumn{2}{|c|}{$\begin{array}{c}\text { tgeom }_{\text {geo }} \\
(\mathrm{min})\end{array}$} \\
\hline & & & PW & SC & PW & SC & PW & $\mathrm{SC}$ & PW & $\mathrm{SC}$ & PW & SC \\
\hline \multirow[t]{4}{*}{ A } & 0,0 & 0,23 & $11,88 \pm 0,08$ & $10,6 \pm 0,10$ & $10,7 \pm 1,27$ & $17,8 \pm 0,41$ & 0,23 & 0,49 & 205 & 58 & 10 & 12 \\
\hline & $-2,5$ & 0,22 & $7,58 \pm 0,29$ & $8,15 \pm 0,09$ & $11,0 \pm 2,33$ & $8,89 \pm 0,71$ & 0,51 & 0,43 & 79 & 128 & 18 & 17 \\
\hline & $-5,0$ & 0,21 & $4,07 \pm 0,35$ & $5,53 \pm 0,12$ & $8,24 \pm 0,30$ & $4,55 \pm 0,13$ & 1,25 & 0,41 & 42 & 208 & 56 & 36 \\
\hline & $-10,0$ & 0,19 & $1,80 \pm 0,07$ & $4,31 \pm 0,08$ & $7,16 \pm 0,07$ & $2,64 \pm 0,06$ & 5,70 & 0,37 & 10 & 344 & 290 & 50 \\
\hline \multirow[t]{4}{*}{ B } & 0,0 & 0,27 & $9,37 \pm 024$ & $8,45 \pm 0,19$ & $10,60 \pm 0,42$ & $14,40 \pm 0,18$ & 0,44 & 0,73 & 131 & 54 & 21 & 26 \\
\hline & $-2,5$ & 0,24 & $5,42 \pm 1,05$ & $6,03 \pm 0,06$ & $7,77 \pm 0,50$ & $6,16 \pm 0,48$ & 0,93 & 0,56 & 76 & 166 & 56 & 43 \\
\hline & $-5,0$ & 0,21 & $4,66 \pm 0,48$ & $4,79 \pm 0,58$ & $4,41 \pm 1,16$ & $3,66 \pm 0,71$ & 0,51 & 0,44 & 190 & 239 & 52 & 50 \\
\hline & $-10,0$ & 0,21 & $2,88 \pm 0,76$ & $4,40 \pm 0,18$ & $5,41 \pm 0,87$ & $2,56 \pm 0,09$ & 1,70 & 0,38 & 56 & 356 & 136 & 63 \\
\hline \multirow[t]{3}{*}{$\mathrm{A} 2 / \mathrm{B}$} & 0,0 & 0,23 & $11,30 \pm 0,06$ & $7,35 \pm 0,43$ & $0,67 \pm 0,01$ & $17,1 \pm 0,55$ & 0,18 & 0,99 & 434 & 32 & 12 & 26 \\
\hline & $-2,5$ & 0,22 & $6,85 \pm 0,22$ & $4,78 \pm 0,16$ & $1,07 \pm 0,53$ & $6,63 \pm 0,50$ & 0,07 & 0,86 & 6369 & 77 & 30 & 55 \\
\hline & $-5,0$ & 0,21 & $5,91 \pm 0,19$ & $6,26 \pm 0,32$ & $5,84 \pm 0,86$ & $4,43 \pm 0,38$ & 0,49 & 0,31 & 161 & 408 & 33 & 28 \\
\hline
\end{tabular}


hidráulica. Os valores de $t_{\text {geom, }}$, muito inferiores a $t_{\text {grav }}$, mostram que o caráter tridimensional imposto pela geometria da fonte ao escoamento resulta no estabelecimento de um regime estacionário muito antes que a gravidade desempenhe um papel importante, possibilitando a realização de experimentos mais rápidos que os tradicionais de infiltração vertical. Isto pode ser verificado na Fig. 2, que representa a evolução do fluxo com o tempo para um conjunto de três repetições na profundidade de $70 \mathrm{~cm}$, com $\psi_{0}=-5 \mathrm{~cm}$, utilizando-se o disco P1. Para este caso, o tempo gravimétrico foi de 208 min e o geométrico de $37 \mathrm{~min}$. O regime estacionário foi alcançado de forma muito rápida $\mathrm{em}$, aproximadamente, $\mathrm{t}_{\mathrm{geom}} / 9$.

A comparação entre os resultados obtidos nas profundidades de 10 e $70 \mathrm{~cm}$, respectivamente nos horizontes A e B, mostram uma diminuição da condutividade menor do que era esperada pelo aumento da fração argilosa. Tal fato pode ter sido causado pela porosidade interagregados, relacionada com a microestrutura do horizonte $\mathrm{B}$, que pode contribuir de maneira importante para o aumento da velocidade de infiltração, como mostram os valores do raio característico de poros.

O comportamento diferenciado do horizonte $\mathrm{A} 2 / \mathrm{B}$, profundidade de $180 \mathrm{~cm}$, pode ser interpretado referindo-se à estrutura já assinalada: 1) nos potenciais menos negativos, de 0 a $-2,5 \mathrm{~cm}$, de fornecimento da água, a porosidade interagregados, isto

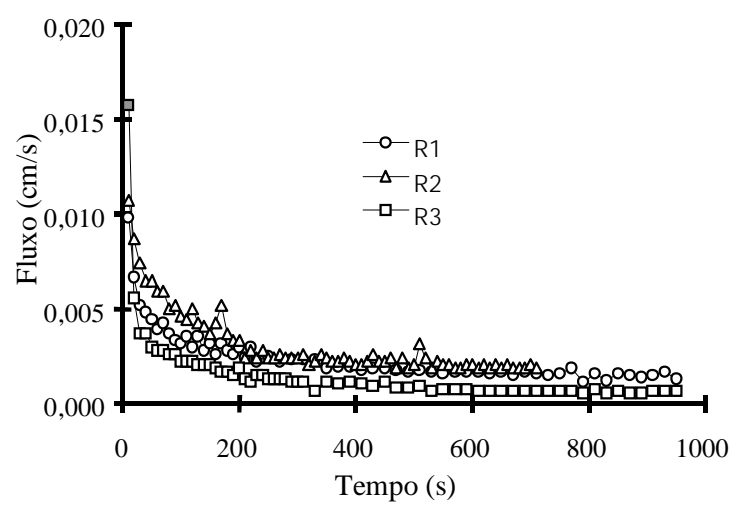

FIG. 2. Evolução do fluxo com o tempo para três repetições (R1, R2 e R3), na profundidade de $70 \mathrm{~cm}$, potencial de fornecimento $-5 \mathrm{~cm}$ e raio do disco permeâmetro de $12,5 \mathrm{~cm}$. é, a rede arenosa, embranquecida e porosa, parece ser também a responsável por uma infiltração aparentemente mais rápida em relação às características granulométricas globais, já que se obteve um maior raio característico de poro e um menor tgrav nessa profundidade; 2) a partir do potencial de fornecimento de água de $-5 \mathrm{~cm}$, pela participação dos volumes argilosos compactados no processo, tem-se uma diminuição importante do raio característico de poros, acarretando aumento da sorvidade e do $t_{\mathrm{grav}}$, a predominância das forças capilares no processo de infiltração, contrariamente ao observado em relação aos potenciais de 0 e $-5 \mathrm{~cm}$, onde as forças gravitacionais apresentam uma maior influência sobre o processo.

\section{CONCLUSÕES}

1. O método de Smettem \& Clothier apresenta melhores resultados nas determinações da sorvidade e da condutividade hidráulica que o método de Perroux \& White.

2. A sorvidade é tão importante quanto a condutividade hidráulica para caracterizar o processo de infiltração.

3. A redução brusca dos valores dos raios característicos de poros do horizonte A2/B, com mudanças no potencial de fornecimento de água, revela a heterogeneidade da estrutura desse horizonte, permitindo identificar a influência dos volumes argilosos compactados sobre o processo de infiltração.

\section{REFERÊNCIAS}

CLOTHIER, B.E.; WHITE, I. Measurement of sorptivity and water diffusivity in the field. Soil Science Society of America. Journal, Madison, v.45, p.241-245, 1981.

COOK, F.J.; BROEREN, A. Six methods for determining sorptivity and hydraulic conductivity with disc permeameters. Soil Science, Baltimore, v.157, p.3-11, 1994.

DIRKSEN, C. Determination of soil water diffusivity by sorptivity measurements. Soil Science Society of America. Proceedings, Madison, v.39, p.22-27, 1975. 
GARDNER, W.R. Some steady-state solutions of unsaturated moisture flow equation with application to evaporation from a water table. Soil Science, Baltimore, v.85, p.228-232, 1958.

PERROUX, K.M.; WHITE, I. Designs for disc permeameters. Soil Science Society of America. Journal, Madison, v.52, p.1205-1215, 1988

PHILIP, J.R. The quasi-linear analysis, the scattering analog, and other aspects of infiltration and seepage. In: FOK, Y.S. (Ed.). Infiltration development and application. Honolulu: Water Resources Research Center, 1987. p.1-27.

PHILIP, J.R. Theory of infiltration. Advances in Hydroscience, San Diego, v.5, p.215-297, 1969.

PHILIP, J.R. The theory of infiltration: 4. Sorptivity and algebraic infiltration equations. Soil Science, Baltimore, v.84, p.257-265, 1957.

REICHARDT, K.D.; NIELSEN; D.R.; BIGGAR, J.W Scaling of horizontal infiltration into homogenous soils. Soil Science Society of America. Proceedings, Madison, v.36, p.241-245, 1972.

SAUER, T.J.; CLOTHIER, B.E.; DANIEL, T.C. Surface measurements of the hydraulic properties of tilled and untilled soil. Soil and Tillage Research, Amsterdam, v.15, p.359-369, 1990.
SMETTEM, K.R.J.; CLOTHIER, B.E. Measuring unsaturated sorptivity and hydraulic conductivity using multiple disc permeameters. Journal of Soil Science, Oxford, v.40, p.563-568, 1989

TALSMA, T. In situ measurements of sorptivity. Australian Journal of Soil Research, Melbourne, v.7, p.269-276, 1969.

THONY, J.L.; VACHAUD, G.; CLOTHIER, B.E.; ANGULO-JARAMILLO, R. Field measurement of the hydraulic properties of soil. Soil Technology, Amsterdam, v.4, p.111-123, 1991

WATSON, K.W.; LUXMOORE, R.J. Estimating macroporosity in a forest watershed by use of a tension infiltrometer. Soil Science Society of America. Journal, Madison, v.50, p.578-582, 1986.

WHITE, I.; SULLY, M.J. Macroscopic and microscopic capillary length and time scales from field infiltration Water Resources Research, v.23, p.1514-1522, Washington, 1987

WOODING, R.A. Steady infiltration from a shallow circular pond. Water Resources Research, Washington, v.4, p.1259-1273, 1968. 